\title{
The importance of synovial adiponectin values in patients with rheumatoid arthritis in comparison with osteoarthritis
}

\author{
Kamila Hashemzadeh $^{1}$, Maryam Sahebari ${ }^{1}$, Mohammad Moeini Nodeh ${ }^{1}$, Zahra Rezaieyazdi ${ }^{1}$, Zhaleh Shariati \\ Sarabi $^{1}$, Zahra Mirfeizi*1
}

\author{
${ }^{1}$ Rheumatic Diseases Research Center (RDRC), Mashhad University of Medical Sciences, Mashhad, Iran
}

\begin{abstract}
This case control study investigated the serum and synovial fluid adiponectin levels in 33 patients with rheumatoid arthritis (RA) and 26 patients with osteoarthritis (OA) as the control group.

A venous blood sample was collected from each patient in fasting condition to measure plasma adiponectin. At the same time, synovial fluid was aspirated aseptically from the swollen knee joint of all patients and controls. Blood and synovial fluid samples were centrifuged at 5,000 rpm for $5 \mathrm{~min}$, then frozen and stored at $-20^{\circ} \mathrm{C}$. For analysis of data, the independent $\mathrm{t}$ test, Mann-Whitney test, Kolmogorov-Smirnov test, Pearson's correlation, and linear regression analysis were utilized.

Serum and synovial fluid adiponectin levels were significantly higher in RA patients than OA subjects $(P$ value $<0.01$ and $P$ value $<0.001$, respectively). In RA patients, serum adiponectin levels were considerably higher than synovial fluid adiponectin levels $(P$ value $<0.001)$. A significant correlation was found between serum and synovial fluid adiponectin levels $(P$ value $<0.001$, $\mathrm{r}=0.63)$ and also between serum adiponectin level and disease duration in RA patients $(P$ value $=0.01, \mathrm{r}=0.42$. Serum and synovial fluid adiponectin were not related to age, body mass index, or disease activity in RA patients.

The present study revealed that adiponectin levels were increased in the serum and synovial fluid of RA patients compared with OA patients, and these levels may have a significant role in the pathogenesis of RA.
\end{abstract}

Keywords: Adiponectin, Rheumatoid arthritis, Synovial fluid, Osteoarthritis

\section{Introduction}

Rheumatoid arthritis (RA) is a chronic systemic autoimmune and destructive disorder characterized by synovial hyperplasia and progressive damage to the articular structure in most patients, which can lead to deformity and dysfunction [1]. Inflammatory cell recruitment and high conf centrations of proinflammatory cytokines in the synovial fluid and circulation of RA patients are some crucial pathogenic causes [2]. Numerous etiologies including genetic and environmental factors are responsible for this disease [3]. As atherosclerosis occurs faster than usual or sooner than expected in RA patients, factors like adipocytokine have been considered in its pathogenesis. Adiponectin is a multifunction cytokine that is secreted from adipocytes. It plays a central metabolic role by its insulin sensitivity and anti-atherogenic effects. On the other hand, the influence of adiponectin on immune response is still controversial because of its pro- and anti-inflammatory properties [3]. Recently, several studies have suggested that adipocytes have the potential to secrete proinflammatory molecules, complement factors, signaling molecules, growth factors, and adhesion molecules [4-7] which can cause tissue in] flammation. Adiponectin may have an important role in the pathogenesis of RA because of the similarity of its two receptors with tumor necrosis factor alpha (TNF_a) $[8,9]$. Serum and synovial levels of adiponectin were ine creased in RA patients. It has been shown that adiponectin has a positive correlation with the serum levels of certain inflammatory cytokines such as VEGF, MMP, TNF-a, IL-1ß, IL-6, and IL-8 which are secreted from synovial fibroblasts because of the proinflammatory effect of adiponectin on creating synovitis and its dissociative effect in the intracellular matrix in RA $[10,11]$. However, the exact role of adiponectin in RA remains unknown because of conflicting reports about its function [10, 12-15].

With regard to the proinflammatory effects of adiponectin, in this study, adiponectin levels in the serum and synovial fluid of RA patients were measured and compared with those of OA patients as the control group. 


\section{Materials and Methods}

\section{Study Subjects}

In this case-control study, all patients who referred to the Rheumatic Diseases Research Center (RDRC), Mashhad University of Medical Sciences, Mashhad, Iran, between Jan., 2012 and Feb., 2013 were examined for eligibility. In total, 33 RA patients diagnosed according to the 1987 ACR criteria who had knee effusion needing therapeutic arthrocentesis were recruited as the study group [12]. The control group comprised 26 patients diagnosed with knee osteoarthritis based on ACR criteria who were candidates for therapeutic arthrocentesis. All participants signed an informed consent form prior to the initiation of the study. The study was approved by the Mashhad University of Medical Sciences Ethics Committee.

A questionnaire collecting data on demographics, drug history, tender and swollen joints count, patients visual analogue scale (VAS), erythrocyte sedimentation rate (ESR), $\mathrm{C}$-reactive protein (CRP), rheumatoid factor (RF), anti-cyclic citrullinated peptide antibody (ACPA), and results of disease activity score 28 (DAS28ESR) were completed for each participant. Exclusion criteria included other connective tissue disorders, any type of coagulopathy, active infection, hypertension, recent ( $<6$ weeks) injection of corticosteroids into the knees, diabetes, body mass index (BMI) $\geq 30 \mathrm{~kg} / \mathrm{m}^{2}$, treatment with insulin, Thiazolidinediones, Telmisartan, Glimepiride, or biologic drugs such as anti TNF- $\alpha$ which can affect adiponectin secretion levels.

\section{Adiponectin Measurement}

A venous blood sample was collected from each patient in fasting condition to measure plasma adiponectin. At the same time, synovial fluid was aspirated aseptically from the swollen knee joint of all patients and controls. Blood and synovial fluid samples were centrifuged at 5,000 rpm for $5 \mathrm{~min}$, then frozen and stored at $-20{ }^{\circ} \mathrm{C}$. Adiponectin levels were detected using ELISA (E09, Mediagnost Company). The inter- and intra-assay coefficients of variability were considered as below $6.7 \%$ and $4.7 \%$, respectively.

The study protocol was reviewed and approved by the local Ethics Committee of Mashhad University of Medical Sciences, and informed consent was obtained from each participant prior to study initiation.

\section{Statistical analysis}

Statistical analysis results are expressed as means \pm standard deviation. Baseline demographics and clinical characteristics were compared between the two groups using the independent $t$ and Mann-Whitney tests. Normality was evaluated using the Kolmogorov-Smirnov test. The correlation coefficient was obtained by Pearson's correlation or the non-parametric Spearman correlation coefficient test wherever appropriate. Linear regression analysis was used to control confounder variables. A value of $P$ value $<0.05$ was considered statistically significant.

\section{Results}

This study was carried out on 33 RA patients, 27 (81.8\%) females and $6(18.2 \%)$ males, and 26 OA patients, 17 (65.4\%) females and $9(25.4 \%)$ males. Mean ages of RA and OA patients were $50.97 \pm 15.18$ and $58.62 \pm 8.23$ years, respectively. The mean age and BMI of OA patients were significantly higher than those of RA patients [ $(P$ val$\mathrm{ue}=0.01, \mathrm{t}=2.46)$ and $(P$ value $=0.005, \mathrm{t}=2.95)$, respectively]. DAS 28 was $6.21 \pm 1.5$ and disease duration was $7.4 \pm$ 6.4 years in RA patients. Table 1 shows the demographic and clinical parameters of patients with RA and OA.

Table 1. Clinical and Demographic Parameters in Patients with RA and OA

\begin{tabular}{|c|c|c|c|}
\hline & $\mathrm{RA}(\mathrm{n}=\mathbf{3 3})$ & $O A(n=26)$ & $P$ value \\
\hline Age (yrs) & $50.97 \pm 15.18$ & $58.62 \pm 8.23$ & 0.01 \\
\hline Female $(\%)$ & 81.8 & 65.4 & 0.1 \\
\hline Mean disease duration (yrs) & $7.4 \pm 6.4$ & $4.7 \pm 5.1$ & 0.07 \\
\hline $\begin{array}{l}\text { Serum } \\
\text { Adiponectin level }(\mu \mathrm{g} / \mathrm{ml})\end{array}$ & $11.62 \pm 6.61$ & $6.43 \pm 3.77$ & $P<0.001$ \\
\hline Synovial fluid Adiponectin level $(\mu \mathrm{g} / \mathrm{ml})$ & $2.87 \pm 2.23$ & $0.42 \pm 0.21$ & $P<0.001$ \\
\hline $\operatorname{ESR}(\mathrm{mm} / \mathrm{h})$ & $37.09 \pm 25.15$ & & \\
\hline DAS-28 ESR & $6.21 \pm 1.5$ & & \\
\hline $\mathrm{BMI}\left(\mathrm{kg} / \mathrm{m}^{2}\right)$ & $23.02 \pm 3.06$ & $25.39 \pm 3.02$ & 0.005 \\
\hline
\end{tabular}


The serum level of adiponectin in patients with RA was $11.62 \pm 6.61 \mu \mathrm{g} / \mathrm{ml}$, while it was $6.43 \pm 3.77 \mu \mathrm{g} / \mathrm{ml}$ in OA patients $(P$ value $<0.01)$. The level of adiponectin in the synovial fluid of RA patients was $2.87 \pm 2.23 \mu \mathrm{g} / \mathrm{ml}$. The same value was $0.42 \pm 0.21 \mu \mathrm{g} / \mathrm{ml}$ in OA patients, indicating a significantly higher level in RA patients $(P$ value $<0.001)$. After omitting the effect of gender, BMI, and disease duration, variance analysis results were still statistically significant. Moreover, serum adiponectin levels in RA patients were significantly higher than synovial fluid adiponectin levels $(P$ value $<0.001)$.

In RA patients, the mean level of serum adiponectin was $10.37 \pm 3.38 \mu \mathrm{g} / \mathrm{ml}$ in males and $11.90 \pm 7.15 \mu \mathrm{g} / \mathrm{ml}$ in females, showing no significant difference regarding gender ( $P$ value $=0.4, \mathrm{t}=0.78)$. Adiponectin levels in the synovial fluid of RA patients were $3.52 \pm 1.25 \mu \mathrm{g} / \mathrm{ml}$ in males and $2.73 \pm 1.25 \mu \mathrm{g} / \mathrm{ml}$ in females, again revealing no meaningful difference $(P$ value $=0.27, \mathrm{t}=1.1)$. In OA patients, the mean level of adiponectin in serum was $6.20 \pm 3.95$ in males and $6.51 \pm 3.79$ in females with no statistical difference between genders $(P$ value $=0.89, \mathrm{t}=0.13)$. In the synovial fluid of OA patients, the adiponectin level was $0.32 \pm 0.16$ among males and $0.47 \pm 0.21$ in females, indicating no meaningful difference based on gender $(P$ value $=0.06, \mathrm{t}=1.9)$. A significantly higher $\mathrm{Sf} /$ serum ratio for adiponectin was found in RA patients compared with the OA group $(P$ value $=0.00)$ (Table 1).

There was a considerable correlation between serum and synovial fluid adiponectin levels in RA patients ( $P$ value $<0.001, \mathrm{r}=0.63$ ); however, such a correlation was not achieved in OA patients (Table 2). In patients with RA, neither serum nor synovial fluid adiponectin levels were correlated with BMI $(P$ value $=0.68, \mathrm{r}=0.07$ and $P$ value $=0.13, \mathrm{r}=0.26$, respectively). The same results were also detected in OA patients (Table 2). However, a significant correlation between serum adiponectin level and disease duration in RA patients was observed when compared with OA controls $(P$ value $=0.01, \mathrm{r}=0.42)$.

The majority of patients with RA in this study had high disease activity measured according to DAS28 (mean: $6.21 \pm 1.5)$. When applying Pearson's correlation analysis, no relationship was detected between disease activity and serum adiponectin level in RA patients $(P$ value $=0.5)$. The results were similar for adiponectin level in synovial fluid $(P$ value $=0.9)$. When RA patients were classified according to DAS activity, again, no correlation was detected between serum and synovial fluid adiponectin levels and mild (DAS28<3.2), moderate ( $<3.2 \mathrm{DAS} 28 \leq 5.1)$, and high disease activity (DAS28>5.1). No significant relationship between adiponectin in either serum $(P$ value $=0.7)$ or synovial fluid $(P$ value $=0.8)$ and serum anti-CCP levels was observed. Furthermore, inflammatory markers such as CRP and ESR were not related to adiponectin levels, in either the serum or the synovial fluid of RA patients (Table 2 ). The correlations between $\mathrm{Sf} /$ serum ratio and clinical/ laboratory manifestations of RA and OA were measured. No correlation was observed between Sf/serum adiponectin ratio and the following variables in OA and RA patients: ESR, CRP, BMI, ACPA, VAS, and DAS 28.

The same correlations were performed once again using regression analysis, and even after omitting the effects of age, gender, and BMI, similar results were achieved.

Table 2. Correlation between serum and synovial fluid adiponectin levels and their clinical and laboratory data in RA and OA patients.

\begin{tabular}{|c|c|c|c|c|}
\hline & \multicolumn{2}{|c|}{ RA } & \multicolumn{2}{|c|}{$\mathbf{O A}$} \\
\hline & Serum adiponectin & Synovial fluid adiponectin & Serum adiponectin & Synovial fluid adiponectin \\
\hline Serum adiponectin & & $P<0.001, \mathrm{r}=0.63$ & & $P=0.47, \mathrm{r}=0.14$ \\
\hline $\begin{array}{l}\text { Synovial fluid adi- } \\
\text { ponectin }\end{array}$ & $P<0.001, \mathrm{r}=0.63$ & & $P=0.47, \mathrm{r}=0.14$ & \\
\hline Disease duration & $\begin{array}{l}P=0.01 \\
\mathrm{R}=0.42\end{array}$ & $\begin{array}{c}P=0.02 \\
\mathrm{R}=0.3\end{array}$ & $P=0.13, \mathrm{r}=0.30$ & $P=0.38, \mathrm{r}=0.17$ \\
\hline BMI & $P=0.68, \mathrm{r}=0.07$ & $P=0.13, \mathrm{r}=0.26$ & $P=0.32, \mathrm{r}=0.20$ & $P=0.84, \mathrm{r}=0.04$ \\
\hline ESR & $P=0.8, \mathrm{r}=0.44$ & $P=0.58, \mathrm{r}=-0.09$ & & \\
\hline CRP & $P=0.4, \mathrm{r}=0.18$ & $P=0.36, \mathrm{r}=0.19$ & & \\
\hline VAS & $P=0.83, \mathrm{r}=-0.03$ & $P=0.47, \mathrm{r}=-0.12$ & & \\
\hline$+\mathrm{ACPA}$ & $P=0.62, \mathrm{r}=-0.1$ & $P=0.83, \mathrm{r}=-0.04$ & & \\
\hline -ACPA & $P=0.82, \mathrm{r}=0.08$ & $P=0.4, \mathrm{r}=-0.31$ & & \\
\hline DAS 28-ESR & $P=0.5, \mathrm{r}=0.12$ & $P=0.9, \mathrm{r}=-0.001$ & & \\
\hline
\end{tabular}

RA: Rheumatoid arthritis; OA: Osteoarthritis: BMI: body mass index; ESR: Erythrocyte sedimentation rate; CRP: C-reactive protein; VAS: visual analogue scale; ACPA: anti citrullinated peptide antibody; DAS-28: disease activity score 28 


\section{Discussion}

The majority of research investigating the role of adiponectin in inflammation has pointed out that the level of this cytokine increases during an inflammatory process [13-17]. However, other studies have reported paradoxical effects of adiponectin on inflammation [18-20]. The presa ent study determined the concentration of adiponectin in matched serum and synovial fluid samples of patients with rheumatoid arthritis and compared them with the same values in individuals with osteoarthritis to evaluate its role in RA. The results demonstrated increased adiponectin levels in the serum and synovial fluid of RA patients compared to OA patients, which is in agreement with most similar studies [13-17]. It was also found that in RA patients, adA iponectin concentration was markedly higher in serum than in synovial fluid. This could be justified by the higher amounts of peripheral fat stores which secrete adiponectin into the blood stream [14].

In accordance with the study of Schaffler et al. [13], the current study found adiponectin levels in the synovial fluid of RA patients were around seven times higher than those in OA patients. However, the serum level of adiponectin in RA patients was twice as high as that of the controls. This difference may be due to the greater production of this factor in synovial fluid than in serum under inflammatory conditions. In vitro studies have shown that under inflammatory conditions, besides fat tissue, adiponectin can be produced by all knee structures, including the infrapatellar adipose tissue, osteophytes, cartilage, and bone [14]. The main cells that are responsible for the secretion of adiponectin are adipocytes, osteoblasts, synovial fibroblasts, and skeletal muscle cells [21-23]. Furthermore, the most important factor in adiponectin secretion is the stimulation of inflammatory cytokines and oxidative stress in those aforementioned parts [14].

Lee et al. demonstrated that adiponectin and IL-1 $\beta$ may act synergistically on the production of proinflammatory mediators such as IL-6, IL-8, and PGE2 in inflammatory arthritic joints [4]. In their recent study, they suggested that the role of adiponectin is more important than that of IL- $1 \beta$ in the production of IL- 6 and IL- 8 in endothelial cells at their physiological concentrations [7].

The assessment of DAS activity in RA patients showed no correlation between adiponectin levels and disease activity; this could be due to the small number of patients in each group.

Corresponding to the present findings, Targonska et al. [10] in 2010 reported a positive correlation between serum and synovial fluid adiponectin levels and disease duration in RA patients. Different factors may have a role in the rise of adiponectin level in long term RA, e.g. rheumatoid cachexia, early atherosclerosis, and progression of the dis- ease [24]. On the other hand, some investigations indicate that the rise in adiponectin level in RA acts as a protecting factor against the atherosclerotic effects of longstanding disease [25-28].

Similar to the current findings, several studies have reported no correlation between adiponectin levels and BMI in RA patients $[14,25,26]$. This may be related to the exs clusion of patients with a BMI $>30$ from the current study. Moreover, certain investigations on different inflammatory and autoimmune diseases have shown no relationship between adiponectin level and BMI [14]. This result can also suggest that the regulation of the serum level of adiponectin is complex, and weight is not the only major regulatory factor.

Nevertheless, no association between adiponectin and systemic inflammatory markers such as CRP or ESR was observed in the current study, which is in agreement with other related studies [14, 15, 21, 22]. Moreover, no correlation between adiponectin levels and ACPA was found in the current study, which may be due to the higher production of adiponectin in synovial fluid rather than serum with a smaller effect on systemic factors.

In their study, Honsawek and Chayanupatkul also measured adiponectin concentrations in plasma and synovial fluid of patients with knee OA and examined the correlation between adiponectin levels and disease severity. They discovered that adiponectin levels in both plasma and synovial fluid were reduced significantly as the severity of OA increased. These findings imply that adiponectin may have a protective role in OA. More studies are needed in order to gain further understanding of the potential utility of adiponectin as a biochemical determinant of disease progression and prognosis [29].

In conclusion, the present study showed higher amounts of adiponectin in systemic circulation compared to synovial fluid. However, its higher level in the synovial fluid of RA patients compared with OA patients suggests that local inflammation may have a role in this elevation.

On the other hand, its high level in the synovial fluid of RA patients might be due to the high serum levels and disease activity. Therefore, it could not be proven with certainty that adiponectin is a marker of local inflammation in RA. In comparison to OA, this study suggested a higher $\mathrm{Sf} /$ serum ratio of adiponectin in RA samples. That finding could be a consequence of higher inflammation or the inflammatory role of adiponectin in RA.

To date, different clinical and experimental studies have been performed on adiponectin activity, and our knowledge regarding this factor is increasing. A better understanding of the genetic basis of adiponectin in different diseases and the cellular events developing in these conditions can enhance our knowledge leading to the administration of new 
methods and better treatment of such cases.

Larger studies are still needed to further clarify the potential influence of adiponectin on disease outcome.

\section{Acknowledgments}

This article was extracted from the thesis prepared by Dr. Mohammad Moeini Nodeh (No.900386) to fulfill the requirements for a Doctorate of Internal Medicine Specialty. The authors would like to thank all of the patients for their kind participation.

\section{References}

1. Giles JT, Allison M, Bingham CO, Scott WM, Bathon JM. Adiponectin is a mediator of the inverse association of adiposity with radiographic damage in rheumatoid arthritis. Arthritis Care \& Research 2009; 61(9):1248-56. doi: 10.1002/art.24789

2. Busso N, So A, Chobaz-Péclat V, Morard C, Martinez-Soria $\mathrm{E}$, Talabot-Ayer D. et al. Leptin signaling deficiency impairs humoral and cellular immune responses and attenuates experimental arthritis. The Journal of Immunology 2002; 168(2):875-82. doi: 10.4049/jimmunol.168.2.875

3. Hulsmans HM, Jacobs JW, Van Der Heijde DM, Albada-Kuipers V, Grietje A, Schenk Y. et al. The course of radiologic damage during the first six years of rheumatoid arthritis. Arthritis \& Rheumutism 2000;43(9):1327-40. 10.1002/1529-0131(200009)43:9<1927:aid-anr3>3.0.co;2-b

4. Coppack SW. Pro-inflammatory cytokines and adipose tissue. Proceedings of the Nutrition Society 2001; 60(03):349-56. doi: 10.1079/pns2001110

5. Chaldakov G, Stankulov I, Hristova M, Ghenev P. Adipobiology of disease: adipokines and adipokine-targeted pharmacology. Current pharmaceutical design 2003; 9(12):1023-31. doi: 10.2174/1381612033455152

6. Ushiyama T, Chano T, Inoue K, Matsusue Y. Cytokine production in the infrapatellar fat pad: another source of cytokines in knee synovial fluids. Annals of the rheumatic diseases 2003; 62(2):108-12. doi: 10.1136/ard.62.2.108

7. Yamasaki S, Nakashima T, Kawakami A, Miyashita T, Tanaka F, Ida H. et al. Cytokines regulate fibroblast-like synovial cell differentiation to adipocyte-like cells. Rheumatology 2004; 43(4):448-52. doi: 10.1093/rheumatology/keh092

8. Yamauchi T, Kamon J, Ito Y, Tsuchida A, Yokomizo T, Kita $\mathrm{S}$. et al. Cloning of adiponectin receptors that mediate antidiabetic metabolic effects. Nature 2003; 423(6941):76269. doi: 10.1038/nature01705

9. Debard C, Laville M, Berbe V, Loizon E, Guillet C, Morio-Liondore B. et al. Expression of key genes of fatty acid oxidation, including adiponectin receptors, in skeletal muscle of Type 2 diabetic patients. Diabetologia 2004; 47(5):917-25. doi: 10.1007/s00125-004-1394-7

10. Targońska-Stępniak B, Dryglewska M, Majdan M. Adiponectin and leptin serum concentrations in patients with

\section{Authors' contributions}

Kamila Hashemzadeh and Zahra Mirfeizi designed and wrote the manuscript. Maryam Sahebari revised some parts of the manuscript. All authors participated in collecting the data. All authors read and approved the final version submitted for publication.

\section{Conflict of interest}

The author confirms that no known conflicts of interest are associated with this article.

rheumatoid arthritis. Rheumatology international 2010; 30(6):731-37. doi: 10.1007/s00296-009-1053-x

11. Neumann E, Frommer K, Vasile M, Müller-Ladner U. Adipocytokines as driving forces in rheumatoid arthritis and related inflammatory diseases? Arthritis \& Rheumatism 2011; 63(5):1159-69. doi: 10.1002/art.30291

12. Arnett FC, Edworthy SM, Bloch DA, Mcshane DJ, Fries JF, Cooper NS. et al. The American Rheumatism Association 1987 revised criteria for the classification of rheumatoid arthritis. Arthritis \& Rheumatism 1988; 31(3):31524. doi: 10.1002/art.1780310302

13. Fowler KB. Adipocytokines in synovial fluid. Jama 2003; 290(13):1709-09.

14. Šenolt L, Pavelka K, Housa D, Haluzik M. Increased adiponectin is negatively linked to the local inflammatory process in patients with rheumatoid arthritis. Cytokine 2006; 35(5):247-52. doi: 10.1016/j.cyto.2006.09.002

15. Ebina K, Fukuhara A, Ando W, Hirao M, Koga T, Oshima K. et al. Serum adiponectin concentrations correlate with severity of rheumatoid arthritis evaluated by extent of joint destruction. Clinical rheumatology 2009; 28(4):445-51. doi: 10.1007/s10067-008-1074-y

16. Tang C-H, Chiu Y-C, Tan T-W, Yang R-S, Fu W-M. Adiponectin enhances IL-6 production in human synovial fibroblast via an AdipoR1 receptor, AMPK, p38, and NF- $\mathrm{kB}$ pathway. The Journal of Immunology 2007; 179(8):548392. doi: 10.4049/jimmunol.179.8.5483

17. Tan W, Wang F, Zhang M, Guo D, Zhang Q, He S: High adiponectin and adiponectin receptor 1 expression in synovial fluids and synovial tissues of patients with rheumatoid arthritis. In: Seminars in arthritis and rheumatism: 2009: Elsevier; 2009: 420-27.

18. Laurberg TB, Frystyk J, Ellingsen T, Hansen IT, Jørgensen A, Tarp U. et al. Plasma adiponectin in patients with active, early, and chronic rheumatoid arthritis who are steroid-and disease-modifying antirheumatic drug-naive compared with patients with osteoarthritis and controls. The Journal of rheumatology 2009; 36(9):1885-91. doi: 10.3899/jrheum.080907

19. Nagashima T, Okubo-Fornbacher H, Aoki Y, Kamata Y, Kimura H, Kamimura T. et al. Increase in plasma levels of adiponectin after administration of anti-tumor necrosis factor agents in patients with rheumatoid arthritis. The 
Journal of rheumatology 2008; 35(5):936-38.

20. Chen T-H, Chen L, Hsieh M-S, Chang C-P, Chou D-T, Tsai $\mathrm{S}-\mathrm{H}$. Evidence for a protective role for adiponectin in osteoarthritis. Biochimica et Biophysica Acta (BBA)-Molecular Basis of Disease 2006; 1762(8):711-18. doi: 10.1016/j. bbadis.2006.06.008

21. Otero M, Lago R, Gomez R, Lago F, Dieguez C, Gomez-Reino J. et al. Changes in plasma levels of fat-derived hormones adiponectin, leptin, resistin and visfatin in patients with rheumatoid arthritis. Annals of the rheumatic diseases 2006; 65(9):1198-201. doi: 10.1136/ ard.2005.046540

22. Frommer KW, Zimmermann B, Meier FM, Schröder D, Heil M, Schäffler A. et al. Adiponectin-mediated changes in effector cells involved in the pathophysiology of rheumatoid arthritis. Arthritis \& Rheumatism 2010; 62(10):2886-99. doi: 10.1002/art.27616

23. Ozgen M, Koca SS, Dagli N, Balin M, Ustundag B, Isik A Serum adiponectin and vaspin levels in rheumatoid arthritis. Archives of medical research 2010; 41(6):457-63. doi: 10.1016/j.arcmed.2010.08.012

24. Shehzad A, Iqbal W, Shehzad O, Lee YS. Adiponectin: regulation of its production and its role in human diseases. Hormones (Athens) 2012; 11(1):8-20. doi: 10.1007/ bf03401534

25. Maury E, Brichard S. Adipokine dysregulation, adipose tissue inflammation and metabolic syndrome. Molecular and cellular endocrinology 2010; 314(1):1-16. doi: 10.1016/j.mce.2009.07.031

26. Lara-Castro C, Luo N, Wallace P, Klein RL, Garvey WT. Adiponectin multimeric complexes and the metabolic syndrome trait cluster. Diabetes 2006; 55(1):249-59. doi: 10.2337/diabetes.55.01.06.db05-1105

27. Okamoto Y, Kihara S, Funahashi T, Matsuzawa Y, Libby P. Adiponectin: a key adipocytokine in metabolic syndrome. Clinical Science 2006; 110(3):267-78. doi: 10.1042/ cs20050182

28. Tsatsanis C, Zacharioudaki V, Androulidaki A, Dermitzaki E, CHARALAMPOPOULOS I, Minas V. et al. Peripheral factors in the metabolic syndrome. Annals of the New York Academy of Sciences 2006; 1083(1):185-95. doi: 10.1196/annals.1367.013 